\title{
Effect of paramagnetic manganese ions doping on frequency and high temperature dependence dielectric response of layered $\mathrm{Na}_{1 \cdot 9} \mathrm{Li}_{0 \cdot 1} \mathrm{Ti}_{3} \mathrm{O}_{7}$ ceramics
}

\author{
DHARMENDRA PAL ${ }^{*}$ and J L PANDEY ${ }^{\dagger}$ \\ Department of Applied Physics, Krishna Girls Engineering College, Kanpur 209 217, India \\ ${ }^{\dagger}$ National Physical Laboratory, New Delhi 110 012, India
}

MS received 20 May 2009; revised 23 July 2009

\begin{abstract}
The manganese doped layered ceramic samples $\left(\mathrm{Na}_{1 \cdot 9} \mathrm{Li}_{0 \cdot 1}\right) \mathrm{Ti}_{3} \mathrm{O}_{7}: X \mathrm{Mn}(0 \cdot 01 \leq X \leq 0 \cdot 1)$ have been prepared using high temperature solid state reaction. The room temperature electron paramagnetic resonance (EPR) investigations exhibit that at lower percentage of doping the substitution of manganese ions occur as $\mathrm{Mn}^{3+}$ at $\mathrm{Ti}^{4+}$ sites, whereas for higher percentage of doping $\mathrm{Mn}^{2+}$ ions occupy the two different interlayer sodium/lithium sites. In both cases, the charge compensation mechanism should operate to maintain the overall charge neutrality of the lattice. The manganese doped derivatives of layered $\mathrm{Na}_{1 \cdot 9} \mathrm{Li}_{0 \cdot 1} \mathrm{Ti}_{3} \mathrm{O}_{7}$ (SLT) ceramics have been investigated through frequency dependence dielectric spectroscopy in this work. The results indicate that the dielectric losses in these ceramics are the collective contribution of electric conduction, dipole orientation and space charge polarization. Smeared peaks in temperature dependence of permittivity plots suggest diffuse nature of high temperature ferroelectric phase transition. The light manganese doping in SLT enhances the dielectric constant. However, manganese doping decreases dielectric loss due to inhibition of domain wall motion, enhances electronhopping conduction, and impedes the interlayer ionic conduction as well. Manganese doping also gives rise to contraction of interlayer space.
\end{abstract}

Keywords. Layered ceramics; solid state reaction; frequency dependence dielectric response.

\section{Introduction}

\subsection{Sodium trititanates}

Yakubovich and Kireev (2003) have refined the crystal structure of $\mathrm{Na}_{2} \mathrm{Ti}_{3} \mathrm{O}_{7}$. They have reported that the crystals are monoclinic with $a=0 \cdot 9133(2) \mathrm{nm}, b=0 \cdot 3806(1) \mathrm{nm}, c=$ $0 \cdot 8566(2) \mathrm{nm}, \quad \beta=101 \cdot 57(3)^{\circ}$ and space group, $P_{21} / m$. The structure of $\mathrm{Na}_{2} \mathrm{Ti}_{3} \mathrm{O}_{7}$ is built up from the blocks of six $\mathrm{TiO}_{6}$ octahedra sharing edges joined by octahedral corners in common layers of composition, $\mathrm{Ti}_{3} \mathrm{O}_{7}{ }^{2-}$. Its lattice is monoclinic. Projection along (010) plane of $\mathrm{Na}_{2} \mathrm{Ti}_{3} \mathrm{O}_{7}$ structure is shown in figure 1(a). Figure 1(b) shows the structure of $\mathrm{Na}_{2} \mathrm{Ti}_{3} \mathrm{O}_{7}$ in projection down the $b$-axis. Bonds to oxygen (large circles) from titanium (small circles) are shown, by means of the lighter circles representing atoms at the level $Y=$ $1 / 4$, the heavier at $Y=3 / 4$. The bonds from sodium (medium circles) to oxygen are drawn as dotted lines, and the unit cell is shown in the outline. The layers are placed together in such a way as to form two different kinds of positions for the sodium

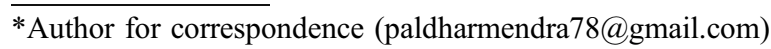

atoms. Na has a rather usual oxygen arrangement, which to a first approximation has been considered as a cube, but with two oxygens replaced by a single one at the centre of the edge, from which they are missing. Outside the faces opposite to this atom are two additional oxygens, which complete the irregular nine-fold coordination. The second sodium atom $\mathrm{Na}_{(1)}$ is situated in a trigonal prism of oxygens, a seventh oxygen is bounded through the centre of one of the rectangular prism faces. The structure of $\mathrm{Na}_{2} \mathrm{Ti}_{3} \mathrm{O}_{7}$ has the possibility of interlayer ionic conduction and can be used as ion exchangers (Izawa et al 1982). Pillaring and photocatalytic properties of partially substituted layered titanates, $\mathrm{Na}_{2} \mathrm{Ti}_{3-x} \mathrm{M}_{x} \mathrm{O}_{7}$ and $\mathrm{K}_{2} \mathrm{Ti}_{4-x} \mathrm{M}_{x} \mathrm{O}_{9}(\mathrm{M}=\mathrm{Mn}, \mathrm{Fe}, \mathrm{Co}, \mathrm{Ni}, \mathrm{Cu})$, have been studied by Machida et al (2000). EPR and mixed electronic ionic conductivity studies of manganese doped and 5\% lithium substituted $\mathrm{K}_{2} \mathrm{Ti}_{4} \mathrm{O}_{9}$ have been reported by Pal and Shripal (2007). Correlation between EPR, dielectric spectroscopic and a.c. conductivity studies have been reported by Pal et al (2006). EPR and electric conductivity studies of copper doped sodium lithium trititanates have also been reported by Pal et al (2005). EPR studies of iron and manganese doped trititanates have been reported by Tangri et al (2005).

In the present paper, we have reported the influence of manganese doping on frequency dependence dielectric 

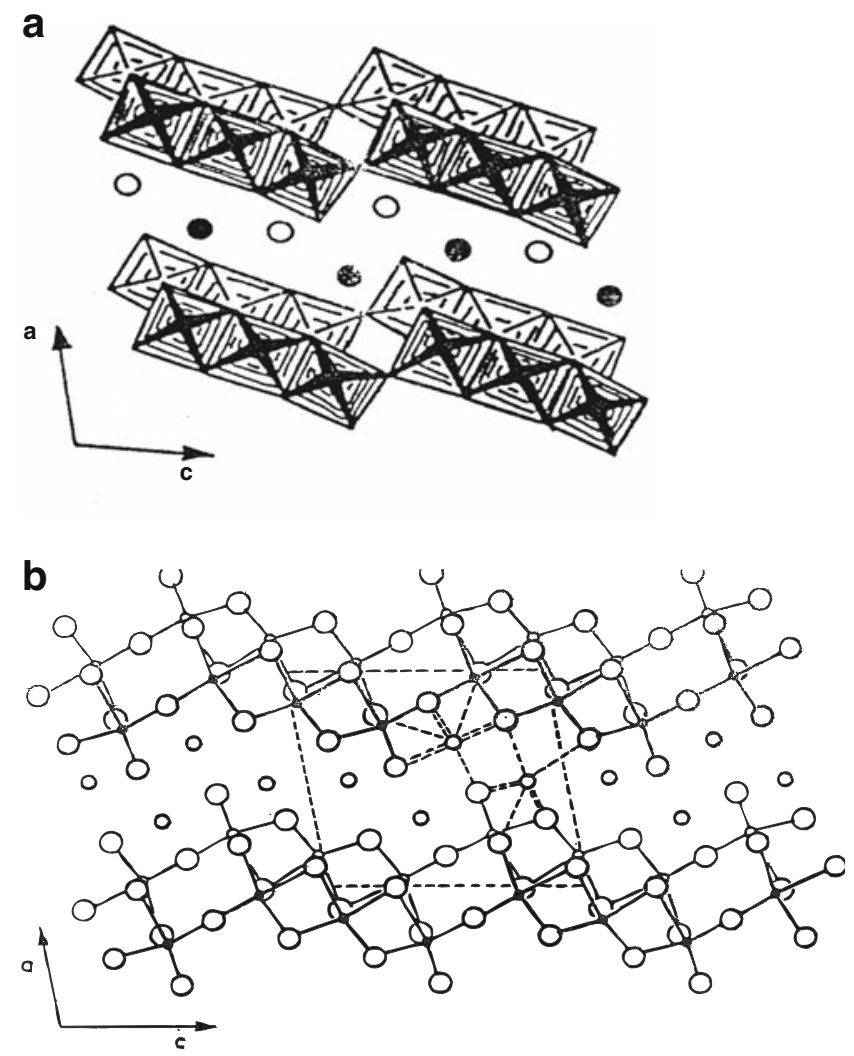

Figure 1. a. Projection along (010) plane of $\mathrm{Na}_{2} \mathrm{Ti}_{3} \mathrm{O}_{7}$ structure and $\mathbf{b}$. structure of $\mathrm{Na}_{2} \mathrm{Ti}_{3} \mathrm{O}_{7}$ seen in projection down the $b$-axis.

response of layered $\mathrm{Na}_{1 \cdot 9} \mathrm{Li}_{0 \cdot 1} \mathrm{Ti}_{3} \mathrm{O}_{7}$ ceramics with the help of EPR studies.

\section{Experimental}

The ceramic sample, $\mathrm{Na}_{1 \cdot 9} \mathrm{Li}_{0 \cdot 1} \mathrm{Ti}_{3} \mathrm{O}_{7}$ (denoted as SLT), and its manganese-doped derivatives with different molar percentages of $\mathrm{MnO}_{2}(0.01$ and $0 \cdot 1)$ (denoted as MSLT-1 and MSLT-2) have been synthesized using the sintering process (Pal et al 2006). The method of preparation of pelletized doped ceramic samples was similar to that reported earlier in the literature (Pal et al 2006). Iso-Debyeflex 2002, Richseifert and $\mathrm{Co}$. diffractometer using $\mathrm{Cu} \mathrm{K} \alpha$ radiation was generated at $30 \mathrm{kV}$ and $20 \mathrm{~mA}$, by collecting XRD patterns for all the compositions. The formation of these titanates (MSLT-1 and MSLT-2) was confirmed by the XRD patterns obtained at room temperature $(R T)$. The XRD pattern of these manganese doped derivatives was identical to that reported in literature (Yakubovich and Kireev 2003). The conventional first derivative of X-band $(9.447 \mathrm{GHz})$ EPR absorption spectra was recorded on a Bruker EMX X-band EPR spectrometer. All the spectra were recorded with $100 \mathrm{kHz}$ and $10.0 \mathrm{G}$ modulations. The maximum calibrated power available was $0 \cdot 201 \mathrm{~mW}$. The high frequency modulation field amplitude ranged typically from $5 \times 10^{-3} \mathrm{mT}-0.50 \mathrm{mT}$ with a rectangular $T E_{102}$ cavity (unloaded $Q \sim 7000$ ) at $100 \mathrm{kHz}$ field modula- tion. Moreover, the samples for recording of the EPR spectra were kept in a quartz tube (outer diameter, $\sim 5 \mathrm{~mm}$ ), which was then placed at the centre of the resonant cavity. An incident microwave power level of $10 \mathrm{~mW}$ had been used for most of the cases to give levels of $10^{5}$. The magnetic field was calibrated using a central field at $3400 \mathrm{G}$.

The flat faces of the sintered pellets were painted with an air-dried high purity silver paste and then mounted in the sample holder evacuated up to $10^{-3}$ mbar for the electrical measurements. The loss tangent $(\tan \delta)$ and parallel capacitance $\left(C_{\mathrm{p}}\right)$ of the pelletized samples were directly measured as a function of temperature and frequency by the HP 4194A impedance analyser. The relative permittivity $\left(\varepsilon^{\prime}\right)$ and the bulk a. c. conductivity $(\sigma)$ of the samples was calculated by using the formula as reported earlier by Pal et al (2006).

\section{Results and discussion}

The $\mathrm{Na}_{2} \mathrm{Ti}_{3} \mathrm{O}_{7}$ crystals are monoclinic with unit cell dimen-

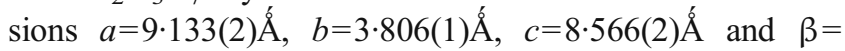
$101 \cdot 57(3)^{\circ}$, space group $=P 2_{1} / \mathrm{m}$. The basic framework of $\mathrm{Na}_{2} \mathrm{Ti}_{3} \mathrm{O}_{7}$ is a block of $\mathrm{TiO}_{6}$ octahedra, three at one level, three at a distance $b / 2 \AA$ above and below which are joined through common edges (Yakubovich and Kireev 2003). The substitution of lithium ions in the interlayer space does not affect the crystal structure of $\mathrm{Na}_{2} \mathrm{Ti}_{3} \mathrm{O}_{7}$. This confirms the presence of small lithium ions with large sodium ions in interlayer space, which indicates that lithium atoms are accommodated with sodium atoms in a widely opened interlayer space.

Figure 2 shows EPR spectra of all the manganese doped derivatives of $\mathrm{Na}_{1 \cdot 9} \mathrm{Li}_{0 \cdot 1} \mathrm{Ti}_{3} \mathrm{O}_{7}$. The room temperature EPR spectra have two peaks, peak ' $A$ ' with $g \approx 4 \cdot 22$ and peak $B$ with $g \approx 2 \cdot 04$. The peak ' $A$ ' in EPR spectrum with a large value of $\mathrm{g}$ indicates that manganese ion substitutes as $\mathrm{Mn}^{3+}$ ( $3 d^{4}$ configuration) at $\mathrm{Ti}^{4+}$ sites and the surrounding oxygen rearranges to yield a tetrahedral field at the ion site, when an abnormal g value may be possible (Abragam and Bleae 1970). It is seen from figure 2 that the intensity of broad peak ' $B$ ' with $g \approx 2 \cdot 04$ increases continuously from sample MSLT-1 to MSLT-2, indicating that the substituting $\mathrm{Mn}^{2+}$ ions in the interlayer space get aggregated and as the doping content of $\mathrm{MnO}_{2}$ increases from MSLT-1 to MSLT-2, major portion of it acquires interlayer cation sites. The fine structure spectrum although very weak consists of five prominent groups of six hyperfine lines each superimposed on broad peak ' $B$ ' with $g \approx 2 \cdot 04$, is indicated for MSLT- 2 .

Figures 3(a) and (b) show the variation of $\tan \delta$ with respect to frequency in the temperature range 373-673 K for MSLT-1 and MSLT-2, respectively. The result shows that at lower temperature $\tan \delta$ decreases gradually, when the frequency increases but at higher temperature dielectric loss $(\tan \delta)$ decreases exponentially with rise in frequency. This may be an indication of dipole mechanism of losses along with the losses due to motion of loosely bound ions, i. e. losses due to electrical conduction (Mac Chesney et al 1963). 


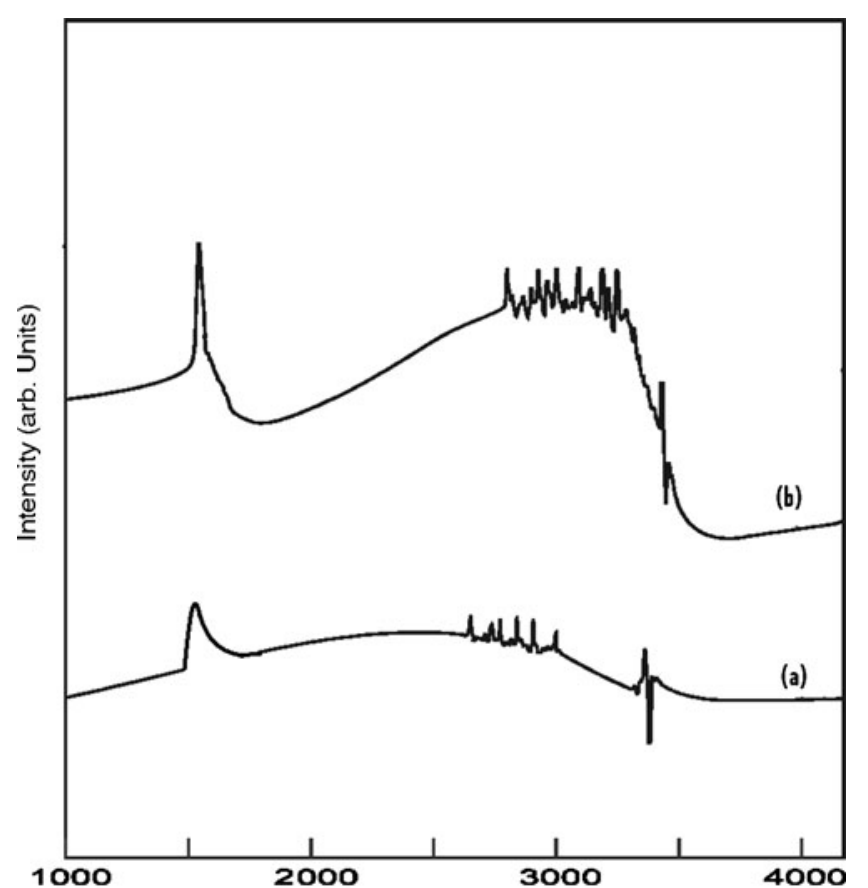

[G]

Figure 2. EPR spectra of manganese doped sodium lithium trititanate (a) MSLT-1 and (b) MSLT-2.
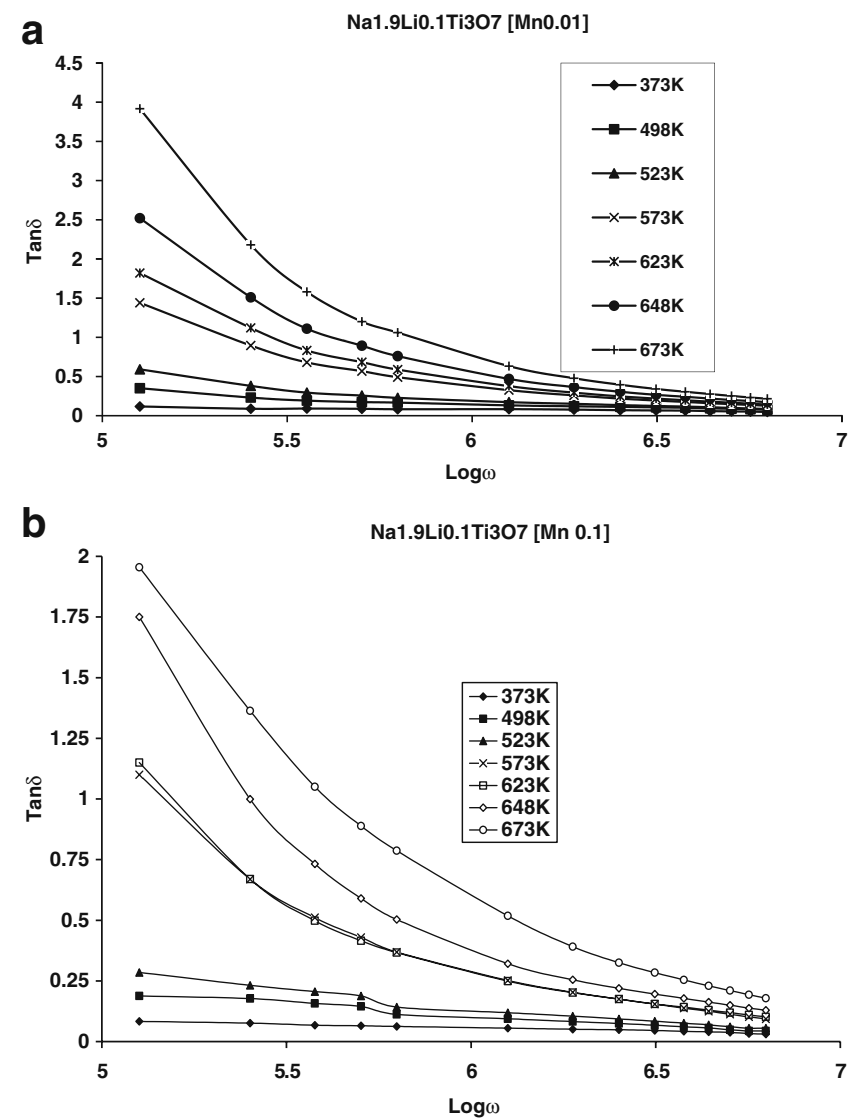

Figure 3. a-b. Loss tangent $(\tan \delta)$ versus frequency for MSLT-1 and MSLT- 2 .
Figures 4(a) and (b) show the variation of dielectric constant $\left(\varepsilon^{\prime}\right)$ versus frequency curves for manganese doped derivatives at various temperatures. The curves have the same nature for MSLT-1 and MSLT-2. The value of dielectric constant $\left(\varepsilon^{\prime}\right)$ is lower at higher frequency. Manganese doping increases the value of dielectric constant $\left(\varepsilon^{\prime}\right)$ for MSLT-1 and MSLT-2. All these curves have same nature as for polar dielectrics. It is reported (Mac Chesney et al 1963) that when the frequency of alternating voltage increases, the value of dielectric constant of polar dielectric remains invariable, but beginning with a certain frequency $\left(f_{0}\right)$, when polarization fails to settle itself during one half period, dielectric constant $\left(\varepsilon^{\prime}\right)$ begins to drop approaching very high frequencies. Dielectric dispersion is seen for these compounds.

$\operatorname{Ln} \sigma T$ versus frequency curves at different temperatures are shown in figures $5\left(a^{\prime}\right)$ and $\left(b^{\prime}\right)$. From these curves, it is clear that the dependence of a.c. conductivity on frequency decreases with increase in temperature. Thus the electronic hopping conduction is dominant at lower temperature and diminishes with the rise in temperature.

EPR spectra of all the manganese doped derivatives show two distinct peaks ' $A$ ' with $g \approx 4 \cdot 22$ and ' $B$ ' with $g \approx 2 \cdot 04$. Peak $A$ with $g \approx 4 \cdot 22$ indicates that lower percentage of $\mathrm{Mn}^{3+}$ doping ( $3 d^{4}$ configuration) substitutes at $\mathrm{Ti}^{4+}$ sites. The broad peak ' $B$ ' with $g \approx 2 \cdot 04$ indicates that at higher concentration of doping manganese, ions enter as $\mathrm{Mn}^{2+}$ at the interlayer alkali
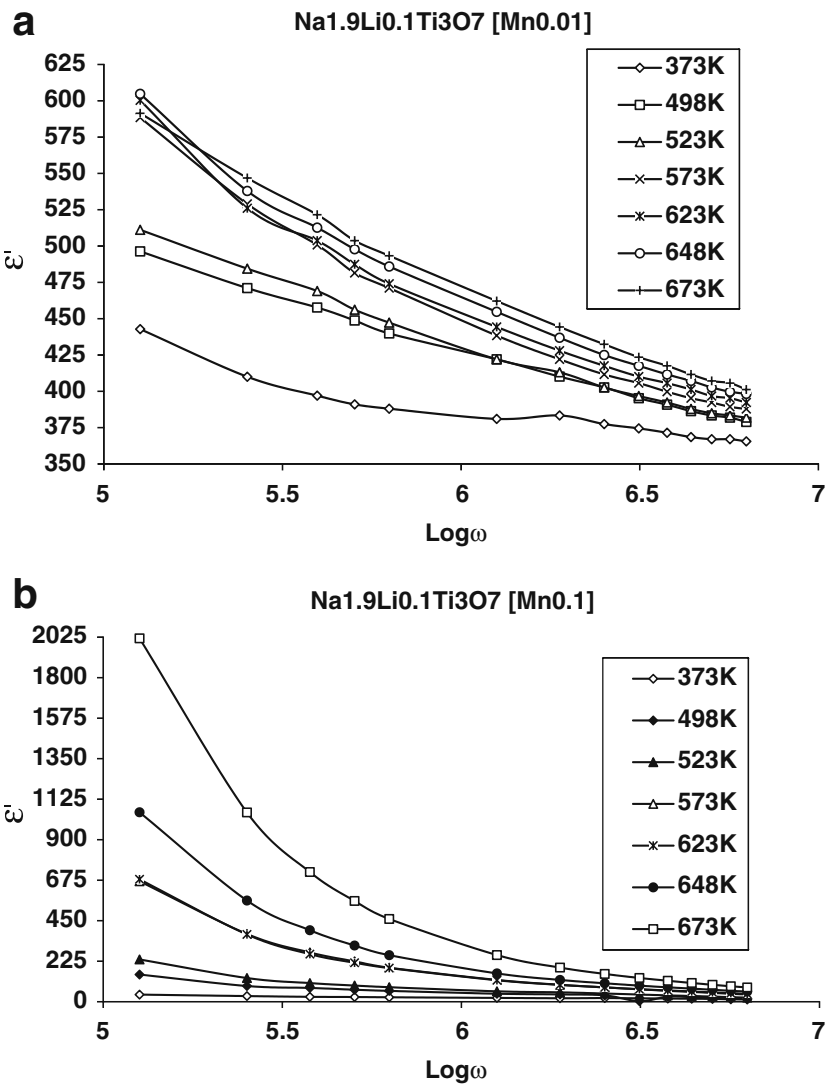

Figure 4. a-b. Relative permittivity versus frequency for MSLT-1 and MSLT-2. 
a

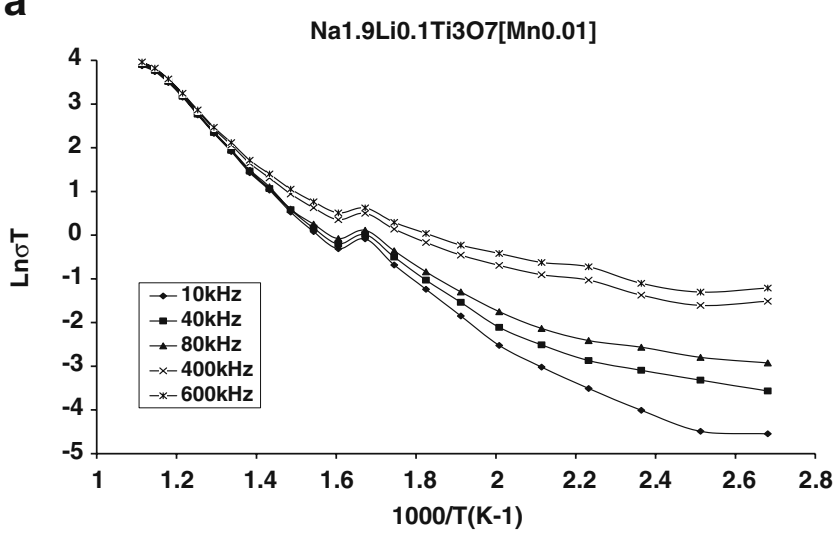

$\mathbf{a}^{\prime}$

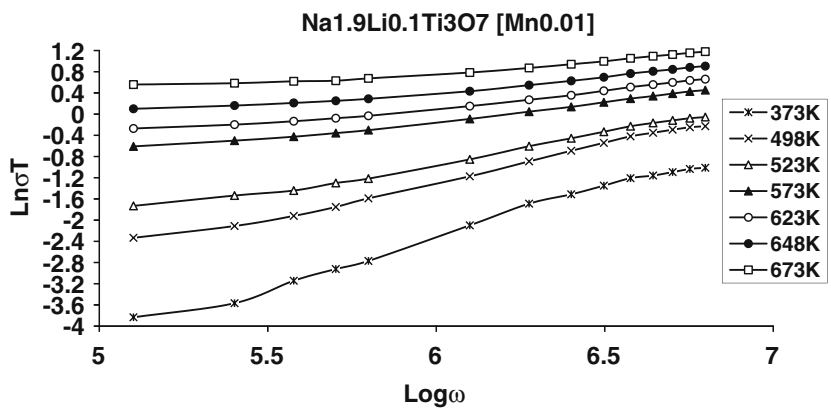

Na1.9Li0.1Ti3O7 [Mn0.1]
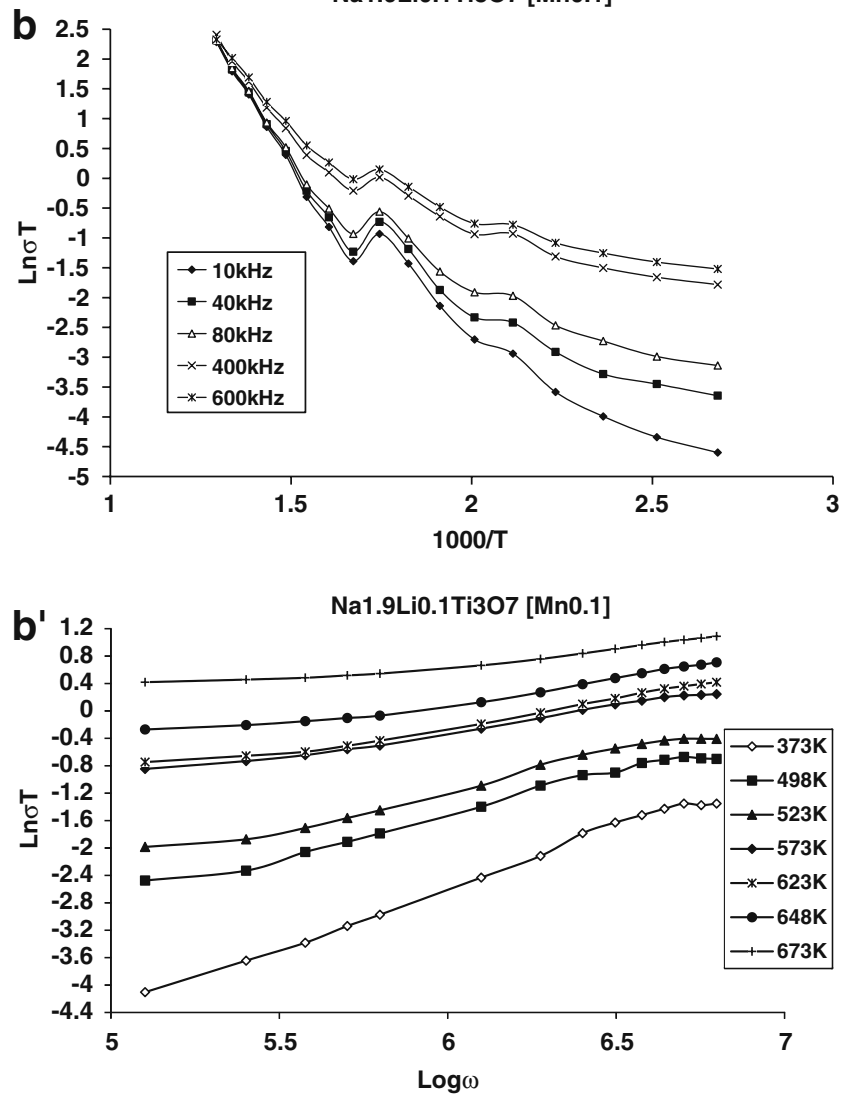

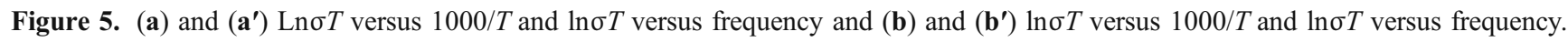

sites, and create the necessary vacancy at the nearby alkali sites. The ion vacancy $(I-V)$ pairs, so obtained would furnish an EPR spectrum consisting of five groups of six lines each. Since the present system is polycrystalline, so the observed EPR spectrum would be averaged as one corresponding to all possible orientations of $I-V$ pairs with respect to the magnetic field. Since we observe only one broad peak at $g \approx 2 \cdot 04$, therefore, an aggregation of $\mathrm{Mn}^{2+}$ ions is proposed, which produces an exchange narrowed EPR signal here.

The dependence of dielectric loss $(\tan \delta)$ on frequency can be explained with the help of equation given below

$$
\operatorname{Tan} \delta=\frac{\omega^{2} \tau^{2}\left(G_{\text {in }}+S\right)+G_{\text {in }}}{\omega\left[S \tau+C_{\mathrm{g}}\left(\omega^{2} \tau^{2}+1\right)\right]},
$$

where the symbols have their usual meanings (Tareev 1979). One can easily explain from this equation

$$
\begin{aligned}
& \operatorname{Lim}_{\cdot \omega \rightarrow 0} \tan \delta=\infty, \\
& \operatorname{Lim}_{\cdot \omega \rightarrow} \infty \tan \delta=0 .
\end{aligned}
$$

The observed appreciable decrease in $\tan \delta$ with rise in frequency at higher temperatures may be due to the accumulation of charges at interfaces in a multiphase material hence interfacial polarization (space charge polarization) takes place (Cook and Tennery 1961; Mac Chesney et al 1963).

The low value of $\tan \delta$ at higher frequencies is the outcome of the low reactance offered by the ceramic sample.
The higher rate of increase of $\tan \delta$ for low frequencies is attributed to the space charge polarization. At higher frequency, samples offer low reactance to the sinusoidal signal and hence minimize the conduction losses, therefore, the amount of dielectric losses are the characteristic of dipole mechanism and electrical conduction.

As the temperature increases from $523 \mathrm{~K}, \tan \delta$ increases sharply, this may be due to its molecular structure with large losses due to the dipole relaxation polarization as the relaxation peaks indicated in the high region. The decreased values of $\tan \delta$ for the samples MSLT- 1 and MSLT-2 suggest that the substitution of $\mathrm{Mn}^{3+}$ at $\mathrm{Ti}^{4+}$ site results in better configuration of the system in terms of losses. Moreover, from these plots it can be seen that there is very little frequency dispersion of dielectric loss. The low value of dielectric loss at high frequency is the outcome of the low reactance offered by the ceramic samples (Lingwal et al 2003). The trend of variation of dielectric loss with frequency is a feature of dielectric loss due to dipole orientation and space charge polarization (Tareev 1979).

The dependence of dielectric constant $\left(\varepsilon_{\mathrm{r}}\right)$ for MSLT-1 and MSLT-2 on frequency at a certain fixed temperature is shown in figures 4(a) and (b). The dependence of $\varepsilon_{\mathrm{r}}$ on frequency has approximately similar nature for samples MSLT-1 and MSLT-2. The values of $\varepsilon_{\mathrm{r}}$ remains invariant up to the temperature $423 \mathrm{~K}$ for MSLT-1 and MSLT-2. The 
existence of three types of dipoles may be due to different roles of three types of dipoles generated due to the substitution of manganese ions $\mathrm{Mn}^{3+}$ at $\mathrm{Ti}^{4+}$ site and $\mathrm{Mn}^{2+}$ at two interlayer alkali sites. It can also be seen that $\varepsilon_{\mathrm{r}}$ continuously decreases as the frequency increases in higher temperature region. The nature of dependence of relative permittivity may be different in solid ionic (linear i.e. not ferroelectric) dielectric. In most of the cases, an ionic mechanism of polarization increases $\varepsilon_{\mathrm{r}}$ when temperature grows. However, in some cases, the values of $\varepsilon_{\mathrm{r}}$ may diminish when temperature rises, particularly in those substances in which the ionic displacement intensifies the internal field and thereby the electronic polarization (Mac Chesney et al 1963). The molecules cannot orient themselves in the polar dielectric in the low temperature region and when temperature raises the orientation of the dipoles is facilitated which increases the dielectric constant. As the temperature grows the chaotic thermal oscillation of the molecules intensifies and degree of orderliness of their orientation diminishes. This causes the curves of dependence $\varepsilon_{\mathrm{r}}$ to pass through the maximum and then drop. The observed dispersion in $\varepsilon_{\mathrm{r}}$ frequency relation can be explained on the basis of Maxwell Wagner model, in which the solid is assumed to be composed of well conducting grains separated by poorly conducting grain boundary. The decrease in dielectric constant with divalent ion doping addition, according to Mac Chesney et al (1963) and Cook and Tennery (1961) was attributed to the formation of weak layers at the grain boundaries of the base matrix, $\mathrm{Na}_{1 \cdot 9} \mathrm{Li}_{0 \cdot 1}$ $\mathrm{Ti}_{3} \mathrm{O}_{7}$. These boundary layers are assumed to be compositionally different from the interior of the grain and may arise because of reaction between $\mathrm{Mn}$ ions and $\mathrm{Na}_{1 \cdot 9} \mathrm{Li}_{0 \cdot 1}$ $\mathrm{Ti}_{3} \mathrm{O}_{7}$ or may originate from $(\mathrm{Mn}-\mathrm{Na} / \mathrm{Li}-\mathrm{Ti})$ complex oxide segregation at the grain boundaries. On manganese doping, dielectric constant increases, the increase in the dielectric constant value is accompanied by the simultaneous rapid decrease in dielectric loss, which may be ascribed to the pinning of domain wall motion due to oxygen vacancies. The acceptor doping activates charge compensation mechanism consequent upon the evolution of oxygen vacancies in the lattice, producing electric dipole constituted by a $\mathrm{Mn}^{2+}$ ion \{effectively negative charge\} and an oxygen vacancy site \{effectively positive charge\}.

Figure 5 shows the $\ln \sigma T\left(\Omega^{-1} \mathrm{~m}^{-1} \mathrm{~K}\right)$ versus $1000 / T$ and $\ln \sigma T\left(\Omega^{-1} \mathrm{~m}^{-1} \mathrm{~K}\right)$ versus $\log \omega$ plots for MSLT-1 and MSLT2 , respectively. These curves are divided into three regions viz. regions I, II and III.

\subsection{Region I (lower temperature region)}

The strongly frequency dependent and almost temperature independent region exists up to $598 \mathrm{~K}$ for MSLT-1 and $573 \mathrm{~K}$ for MSLT-2. In ionic host lattices, where the interaction between orbital of neighbouring ions, there is polarization of lattice associated with the presence of electronic carriers, and its polarization field is referred to as polaron. Two types of polarons play a major role in the conduction process at relatively lower temperature region.
When the association is weak (large polarons), conductivity is similar to quasi-free electrons which results with the small effective mass. When the electronic carrier plus the lattice distortion has the linear dimension smaller than the lattice parameter, it is referred to as a small polaron, and the mobility is strongly affected by the lattice distortion which must move along with the electronic carrier. The loosened electrons from the negative ions and from the impurities in the crystal transported through the solid would contribute to the conduction. The polaron is an electron that always moves around together with the associated lattice polarization that minimizes its energy. The nature of a. c. conductivity can be interpreted by proposing that the electronic hopping conduction, in which the hopping of electrons through shallow barrier along Ti-Ti chain takes part in conduction. These electrons play a major role in this region. Such a frequency dependence of conductivity is attributed to a wide distribution of relaxation times due to barrier height. The conduction mechanism for the low temperature region can be expressed by the equation

$$
\sigma=\sigma_{0} \exp \left[\left(-T_{0} / T\right)^{1 / 4}\right]
$$

\subsection{Region II (mid temperature region)}

This region exists from $598 \mathrm{~K}$ to $623 \mathrm{~K}$ for MSLT- 1 and $573 \mathrm{~K}$ to $598 \mathrm{~K}$ for MSLT-2. It is seen that in this temperature region the conductivity value decreased with increasing temperature. The conduction mechanism in this region has been formulated by proposing that the loose oxygens produced by the substitution of $\mathrm{Mn}^{3+}$ at $\mathrm{Ti}^{4+}$ will be trapped by cation vacancies present in the interlayer space leading to decrease in the conductivity. It suggests that the electronic hopping conduction is still strongly contributing up to this higher temperature region for MSLT-1 and MSLT-2.

So the conduction mechanism for MSLT-1 and MSLT-2 may be hindered by interlayer ionic conduction and electronic hopping conduction.

\subsection{Region III (higher temperature region)}

Almost temperature dependent and very less frequency dependent regions exist from $623 \mathrm{~K}$ for MSLT-1 and from $598 \mathrm{~K}$ for MSLT-2 up to the temperature range of study. The dependence of conductivity upon the frequency persists for manganese-doped derivatives for MSLT-1 and MSLT-2. The electronic contribution is less than the total conduction in the higher temperature region showing that at higher temperatures the conduction is mainly ionic. Accordingly, the conduction mechanism in this region may modify interlayer ionic conduction along with the polaronic conduction for MSLT-1 and MSLT-2. From these curves, it is clear that the dependence of a.c. conductivity decreases with increase in temperature. The frequency dependence of conductivity is the characteristic of electron hopping conduction, which decreases with rise in 
temperature, indicating a decrease in electron hopping conduction and an increase in interlayer ionic conduction.

\section{Conclusions}

(I) Manganese doped layered sodium lithium trititanates, $\mathrm{Na}_{1.9} \mathrm{Li}_{0 \cdot 1} \mathrm{Ti}_{3} \mathrm{O}_{7}$ ceramics, can be put in the class of mixed ionic-electronic materials.

(II) Manganese doping is found to increase the dielectric constant along with the decrease in dielectric loss in lightly doped composition.

(III) Two types of manganese substitutions and dipoles in the interlayer space as well have been identified through tan $\delta$ versus temperature plots for doped derivatives of sodium lithium trititanates $\left(\mathrm{Na}_{1 \cdot 9} \mathrm{Li}_{0 \cdot 1} \mathrm{Ti}_{3} \mathrm{O}_{7}\right)$ ceramic.

(IV) Dielectric-spectroscopic investigations show the presence of dipole mechanism of losses along with the losses due to the motion of loosely bound ions and space charge polarization at higher temperature.

\section{References}

Abragam A and Bleae B 1970 Electron paramagnetic resonance of transition ions (Oxford: Clarendon Press)

Cook R L and Tennery V J 1961 J. Am. Ceram. Soc. 44187

Izawa H, Kikkawa S and Koizumi M 1982 J. Phys. Chem. 86 5023

Lingwal V, Semal B S and Panwar N S 2003 Bull. Mater. Sci. 26 619

Mac Chesney J B et al 1963 J. Am. Ceram. Soc. 46197

Machida Masato, Wei Ma Xu, Taniguchi Hideki, Yabunaka Junichi and Kijima Tsuyoshi 2000 J. Mol. Catal. A: Chemical 155 131

Pal D and Shripal 2007 J. Mater. Sci.: Mater. Electron. 18401

Pal D, Prem Chand, Tandon R P and Shripal 2005 J. Korean Chem. Soc. 49560

Pal D, Tandon R P and Shripal 2006 Indian J. Pure Appl. Phys. 44 435

Tangri Alka, Mishra Pradeep and Pal D 2005 Int. J. Chem. Sci. 3 715

Tareev B 1979 Physics of dielectric materials (Moscow: Mir Publishers) Ch. 3

Yakubovich O V and Kireev V V 2003 J. Crystallogr. Rep. 4824 